\title{
Pharmaceutical Equivalence and Bioequivalence of Radiopharmaceuticals: Thinking the Possibility of Generic Radiopharmaceuticals and Preparing for New Technology as Nanotechnology Drugs
}

\section{Ralph Santos-Oliveira*}

Brazilian Association of Radiopharmacy, Laboratory of Nanoradiopharmaceuticals, Rio de Janeiro, Brazil

\section{Letter}

The use of radiopharmaceuticals has been growing exponentially throughout the world, achieving notable numbers. Recent data estimates that in 2015 the market of Nuclear Medicine and Radiopharmacy surpass the mark of 1.5 trillion dollars, with more than 100 million people making use of these drugs around the world. In this scenario, so overwhelming, is surprising that there has not begun studies of pharmaceutical equivalence and bioequivalence of such drugs, especially with animal model and for therapeutics uses.

Pharmaceutical equivalents is understood as drugs that have the same dosage form, route of administration and the same amount of the same active substance, that is, the same salt or ester of therapeutic molecule, or may contain excipients not identical, since well-established intended function. Equivalent pharmaceuticals, must comply with the same requirements of the Pharmacopoeia monograph, preferably with or other official compendia, rules or regulations adopted or, in their absence, with other standards of quality.

Bioequivalence, in order hand, is used to demonstrate pharmaceutical equivalence between products which have the same pharmaceutical form, containing the same qualitative and quantitative composition of principle(s) active(s), and have comparable bioavailability when studied under the same experimental design.

Obviously there must be considered the various existing laws around the world that deal with radiopharmaceuticals, however, this factor can not be individually responsible for the absence of such studies. The availability of generic radiopharmaceuticals should be viewed as a reality, whether by producers, either by the inspectors. The current situation of lack of such generics on the market, should not be a limiting factor for the future development of such reality. Meanwhile, the advance of new technologies, especially nanotechnology and hence nanoradiopharmaceuticals, become each day closer to becoming reality, and thus, both the concepts of pharmaceutical equivalence and bioavailability should already be updated so that they address this class of drugs, as well as radiopharmaceuticals and thus not be a legal.

In a pioneering and sustained by the poignant need to decrease costs related to nuclear medicine procedures, this letter proposes the initiation of studies in the direction of generic-radiopharmaceuticals and thus increase access and quality of procedures in radiopharmacy and nuclear medicine globally.
*Corresponding author: Ralph Santos-Oliveira, Brazilian Association of Radiopharmacy, Laboratory of Nanoradiopharmaceuticals, Av. Manuel Caldeira de Alvarenga - UEZO - Campo Grande, Rio de Janeiro, Brazil, Tel: +552121733964; E-mail: roliveira@ien.gov.br

Received November 04, 2013; Accepted January 23, 2014; Published January 30, 2014

Citation: Santos-Oliveira R (2014) Pharmaceutical Equivalence and Bioequivalence of Radiopharmaceuticals: Thinking the Possibility of Generic Radiopharmaceuticals and Preparing for New Technology as Nanotechnology Drugs. J Bioequiv Availab 6: 023-023. doi:10.4172/jbb.1000175

Copyright: (c) 2014 Santos-Oliveira R. This is an open-access article distributed under the terms of the Creative Commons Attribution License, which permits unrestricted use, distribution, and reproduction in any medium, provided the original author and source are credited. 